\title{
Oximetría cerebral no invasiva: su uso en cirugía laparoscópica de un lactante
}

\author{
Mena M. ${ }^{1,2}$, Selamé R. ${ }^{1,2}$, Corral G. ${ }^{1,2}$, Córdova S. ${ }^{1,2}$, Araya C. ${ }^{1,2}$ \\ 1 Hospital Gustavo Fricke, Viña del Mar, Chile. \\ 2 Universidad de Valparaíso, Viña del Mar, Chile.
}

Introducción: La oximetría cerebral no invasiva utilizando la tecnología NIRS (near infrared spectroscopy) es útil para detectar isquemia-hipoxia cerebral relacionada con hipoperfusión e hipoxemia y determinar distintas intervenciones para prevenir o disminuir la injuria por hipoxia cerebral.

Uno de los factores involucrados en la injuria cerebral relacionada a la perfusión en el paciente prematuro es atribuida a la falta de autorregulación cerebrovascular.

Aumentos de la presión intrabdominal se han asociado a aumentos de la presión intracranial con la consiguiente disminución de la presión de perfusión cerebral.

Método: Paciente de 7 meses, que nació a las 36 semanas, con antecedentes de coartación aórtica y CIV operada, traqueomalacia severa, traqueostomizado, ingresa a pabellón para realizar cirugía de Nissen laparoscópico y gastrostomía percutánea por los diagnósticos de trastorno de deglución y reflujo gastroesofágico severo.

La monitorización estándar incluyó: saturometría de O2 (SpO2), presión arterial no invasiva (PANI), electrocardiograma, temperatura y $\mathrm{CO} 2$ espirado (etCO2).

Previo a la inducción anestésica se colocaron los sensores de NIRS en la región frontoparietal izquierda y derecha, midiéndose la SrO2C. El valor basal obtenido fue 90\% izquierda/95\% derecha. La PANI basal fue de $65 \mathrm{~mm} \mathrm{Hg}$.

Para la inducción y mantención anestésica se utilizó sevoflurano, suplementado con fentanilo y atracurio.

La ventilación mecánica controlada fue iniciada con un etCO2 basal de $50 \mathrm{mmHg}$. Se inicia la cirugía por vía laparoscópica, partiendo con neumoperitoneo de $10 \mathrm{mmHg}$, sin embargo, durante los siguientes 20 minutos se observa una importante caída de la $\mathrm{SrO} 2 \mathrm{c} 55 \%$ izquieda $/ 52 \%$ derecha, asociándose a aumento del etCO2 de 80 $\mathrm{mmHg}$, sin deterioro de la PAM ni SpO2, se decide desinflar neumoperitoneo, recuperándose la $\mathrm{SrO} 2 \mathrm{c}$ y disminuyendo el etCO2, luego de su recuperación se vuelve a insuflar $\mathrm{CO} 2$ a presión de $6 \mathrm{mmHg}$. Tolera relativamente esta nueva presión, pero tiene una nueva caída de la Sro2c $50 \%$ izquierda $/ 65 \%$ derecha por lo que se decide cambiar a técnica abierta. No presentando nuevos episodios hasta el final de la cirugía.

Resultado y Discusión: La SrO2c medida utilizando la tecnología NIRS es un indicador indirecto de flujo sanguíneo cerebral (FSC) y perfusión cerebral. La Elevación de la presión intraabdominal esta relacionada con aumento de la presión intracraneal con el consiguiente deterioro de la perfusión cerebral. Los neonatos y lactantes son particularmente vulnerables.

El presente caso nos ilustra el potencial valor del uso de NIRS en el ajuste de los parámetros hemodinámicos y ventilatorios y en diagnosticar y sugerir al equipo quirúrgico cambios necesarios en el curso de la cirugía.

https://doi.org/10.25237/congresoclasa2019.26 